\title{
Ferroelectric nanoparticles in low refractive index liquid crystals for strong electro-optic response
}

M. Kaczmarek, O. Buchnev, and I. Nandhakumar

School of Chemistry, University of Southampton, Southampton UK SO17 1BJ. Email: iris@soton.ac.uk

Please cite this paper as:

Applied Physics Letters, 2008, (92) 103307

The publisher's version of this paper is available here:

http://dx.doi.org/10.1063/1.2884186

\section{Related articles by Dr Iris Nandhakumar can be found below:}

X.Li, G.S.Attard M. Markham, D. C. Smith, J.J. Baumberg and I.S. Nandhakumar, (2009) Nanotemplated lead telluride thin films, Microporous and Mesoporous Materials, 118, (1-3), 403-407 (2009). (doi:10.1016/j.micromeso.2008.09.019).

I.S. Nandhakumar, T.Gabriel, X.Li, M. Markham, D.C. Smith, J.J. Baumberg, G.S. Attard, (2006) Electrodeposition of Mesoporous CdTe Films with the aid of Citric Acid from Lyotropic Liquid Crystalline Phases, J. Mat. Sci., 16 (31): 3207-3214 (doi:10.1039/b603911a).

I.S. Nandhakumar, T.Gabriel, X.Li, M. Markham, D.C. Smith, J.J. Baumberg, G.S. Attard, (2004) Optical properties of mesoporous II-VI semiconductor compound films, Chemical Communications, Vol. 12, 1374-1375. (doi:10.1039/b403423f).

T.Gabriel, I. Nandhakumar, G. S. Attard, (2002) Electrochemical synthesis of nanostructured tellurium films, Electrochem. Comm., 4, 610-612. (doi:10.1016/S1388-2481(02)00390-9). 


\title{
Ferroelectric nanoparticles in low refractive index liquid crystals for strong electro-optic response
}

\author{
M. Kaczmarek, ${ }^{1, a)}$ O. Buchnev, ${ }^{1}$ and I. Nandhakumar ${ }^{2}$ \\ ${ }^{1}$ School of Physics and Astronomy, University of Southampton, Southampton SO17 1BJ, United Kingdom \\ ${ }^{2}$ School of Chemistry, University of Southampton, Southampton SO17 1BJ, United Kingdom
}

(Received 19 December 2007; accepted 23 January 2008; published online 11 March 2008)

\begin{abstract}
Functional materials based on ferroelectric, inorganic nanoparticles, and low refractive index nematic liquid crystals show strong induced birefringence and dielectric anisotropy. Birefringence can increase by a factor of 2 and dielectric anisotropy by a factor of 3 as compared with nominally pure liquid crystals. The enhancement of the electro-optic performance is higher in liquid crystals with $\mathrm{Sn}_{2} \mathrm{P}_{2} \mathrm{~S}_{6}$ (SPS) nanoparticles than with $\mathrm{BaTiO}_{3}$ nanoparticles. The shape and size distribution of both types of ferroelectric particles were characterized using atomic force microscopy. The average size of SPS nanoparticles was $45 \mathrm{~nm}$ and of $\mathrm{BaTiO}_{3}$ nanoparticles was $20 \mathrm{~nm}$. (C) 2008 American Institute of Physics. [DOI: 10.1063/1.2884186]
\end{abstract}

New functional optical systems increasingly rely on combining organic and inorganic materials. Hybrid liquid crystal-inorganic systems include, for example, semiconductor nanorods dispersed in liquid crystals for strong reorientational effect ${ }^{1}$ and tunable refractive index. ${ }^{2}$ Semiconductor nanoparticles added to liquid crystals ${ }^{3}$ or polymers can lead to large electro-optic effect ${ }^{4}$ or photorefractive effect. ${ }^{5,6}$

Another example of hybrid materials are the suspensions of ferroelectric nanoparticles ${ }^{7}$ in nematic liquid crystals. Liquid crystals functionalized in this way show increased phase transition temperatures as well as dielectric and optical anisotropy ${ }^{8}$ and have potential applications in, for example, displays $^{9}$ as well as for photorefractive systems. ${ }^{10}$ In this study, we explored the impact of nanoparticles on liquid crystals most suitable for optoelectronic applications, namely, low refractive index liquid crystals. They were synthesized to be index matched to silica with the aim of developing their applications in fiber and waveguide devices. However, low refractive index liquid crystals also have low birefringence $(\Delta n=0.04-0.05)$ and, so far, it proved impossible to improve that parameter via synthethic methods. In this study, two different types of ferroelectric and photorefractive nanocrystals $\left[\mathrm{Sn}_{2} \mathrm{P}_{2} \mathrm{~S}_{6}\right.$ (SPS) and $\left.\mathrm{BaTiO}_{3}\right]$ were used to functionalize low refractive liquid crystals, Merck 18523 and Likchem LC 1550 with the aim of optimizing their dielectric and optical anisotropy.

The first stage of our investigation was devoted to atomic force microscopy (AFM) measurements of the shape, size, and distribution of nanocrystals used for functionalization of liquid crystals, as to the best of our knowledge, such parameters have not been published yet. The nanoparticles were prepared by milling microparticles of SPS, and then separately $\mathrm{BaTiO}_{3},(\cong 1 \mu \mathrm{m}$ size $)$, with oleic acid for $100 \mathrm{~h}$. The expected size of nanoparticles was of the order of $10 \mathrm{~nm}$, based on the published transmission electron microscopy (TEM) images of SPS powder after $25-75 \mathrm{~h}$ of milling. ${ }^{11}$ However, while those TEM images allowed some important size characterization of nanoparticles, their shape and distribution was difficult to resolve in detail. Recently, high-resolution images of $\mathrm{BaTiO}_{3}$ nanocrystals, using AFM

\footnotetext{
a)Electronic mail: mfk@soton.ac.uk.
}

in tapping mode, were published ${ }^{12}$ and, therefore, in our analysis, we opted for this technique. One of the difficulties in obtaining high-resolution images by either TEM, scanning electron microscopy, or AFM is the presence of oleic acid on nanoparticles. While the process of dialysis, ${ }^{13}$ can help purify the suspensions of nanocrystals in solvents, it will not completely remove the surfactant attached to nanocrystals.

In preparation for AFM, SPS and $\mathrm{BaTiO}_{3}$ nanoparticles were deposited on specially prepared silicon substrates. For the preparation of individual $\mathrm{BaTiO}_{3}$ nanocrystals, dilute solutions of $\mathrm{BaTiO}_{3}$ nanoparticles dispersed in hexane (1:2-1:16 by volume relative to an original concentration) were spin coated at high spin rates (4000 rpm) onto $n$-silicon wafers with a $500 \mathrm{~nm}$ thick oxide layer. The $\mathrm{BaTiO}_{3}$ nanocrystals were then characterized by Multimode AFM Veeco in a tapping mode. In order to analyze the size and height distribution of individual nanoparticles, NANOSCOPE and IMAGE SXM softwares were used.

Figure 1(a) presents typical AFM images of $\mathrm{BaTiO}_{3}$ nanoparticles, while Fig. 1(b) shows AFM images of SPS nanoparticles, capturing images of individual, well-separated particles.

The well-resolved AFM images allowed us to analyze the shape and symmetry of the nanoparticles. Both $\mathrm{BaTiO}_{3}$ and SPS nanoparticles have ellipsoid-like shape with SPS being more elongated. The average dimensions of $\mathrm{BaTiO}_{3}$ particles were length of $20 \mathrm{~nm}$ and an average diameter of $15 \mathrm{~nm}$. In the case of SPS, the nanoparticles had an average length of $45 \mathrm{~nm}$ and diameter of $25 \mathrm{~nm}$. These dimensions have an error of $\pm 5 \mathrm{~nm}$. It was interesting to note that, most likely, milling of microcrystals leads to their ellipsoidal shape. The distribution in size of both types of nanoparticles was also analyzed. In case of $\mathrm{BaTiO}_{3}$, the spread in size was confined between $5-40 \mathrm{~nm}$ with the peak diameter being approximately at $15-20 \mathrm{~nm}$. However, SPS nanoparticles turned out to have their sizes spread over larger range, uniformly distributed between $5-80 \mathrm{~nm}$.

The nanoparticles were then added to liquid crystals Merck 18523 and LC1550 at a weight ratio 1:100, ultrasonically mixed for $5 \mathrm{~min}$ with following evaporation of the heptane. Liquid crystals functionalized in this way were then used to infiltrate the cells. The liquid crystal cells were as- 


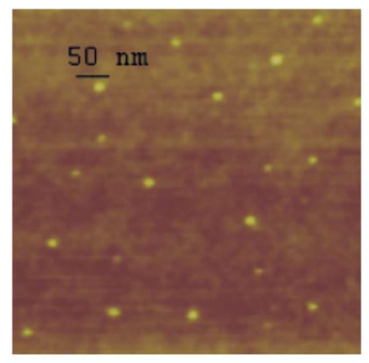

(a)

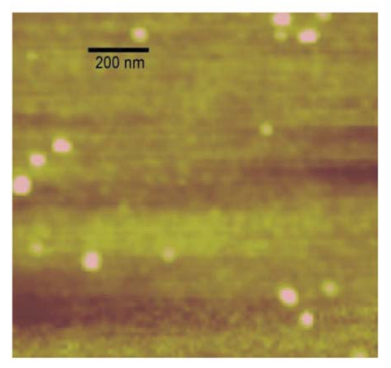

(b)

FIG. 1. (Color online) AFM images of (a) $\mathrm{BaTiO}_{3}$ and (b) SPS nanoparticles.

sembled earlier using two glass substrates, each covered by transparent indium tin oxide electrodes and by $100 \mathrm{~nm}$ polyimide layers. To achieve a homogeneous planar alignment of liquid crystal, both polymer layers were unidirectionaly rubbed with velvet. The cells' thickness was $12 \mu \mathrm{m}$.

In the second stage of this study, birefringence, dielectric anisotropy, reorientation threshold, as well as the phase transition temperatures were determined. The cells were illuminated by a single beam with ac field applied and put between crossed polarizers, placed at $45^{\circ}$ with respect to the liquid crystal director. The transmitted light was collected on a photodiode placed behind the cell and the variation in intensity measured for increasing ac voltage. Figure 2 presents the comparison between results for nominally pure LC 18523, LC $18523+\mathrm{BaTiO}_{3}$ nanoparticles, and LC 18523+SPS nanoparticles. Similar dependence was measured for LC 1550. This figure demonstrates the increased birefringence of suspensions of ferroelectric SPS particles in liquid crystals by inducing larger phase retardation in comparison with both pure liquid crystal as well as those with suspensions of $\mathrm{BaTiO}_{3}$ nanoparticles. The results of birefringence measurements and calculations ${ }^{14}$ are shown in Table I. Furthermore, the suspensions have lower, up to a factor of 2, reorientation threshold voltage as compared with pure liquid crystals, the result of their increased dielectric anisotropy.

Investigating the dielectric properties, the impedance of the cell was measured to estimate the dielectric permittivity of the samples, using standard auto balancing bridge method at the frequency of $10^{3} \mathrm{~Hz}$. The experimental setup was calibrated by the prior measurements of the empty cells.

For completeness, the possible change in phase transition temperatures and pretilt angles were also investigated. ${ }^{15}$ No change in pretilt angles was observed as compared with pure liquid crystals, but an increase in nematic to isotropic phase transition temperature was measured. Using a hot stage and a polarizing microscope, changes in liquid crystal structure could be detected. The samples' temperature was controlled with the precision of $0.05^{\circ} \mathrm{C}$.

Author complimentary copy. Redistribution subject to AIP license or copyright, see http://apl.aip.org/apl/copyright.jsp

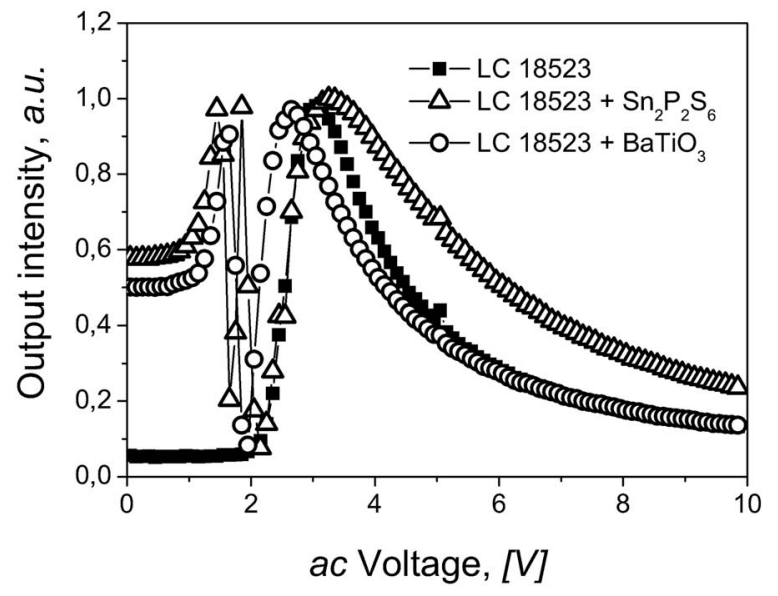

FIG. 2. Transmitted intensity versus ac voltage measured in a polarizer-cellanalyzer setup for pure liquid crystals and liquid crystals with ferroelectric nanoparticles.

Table I summarizes the improvements in all the measured parameters in liquid crystals with ferroelectric nanoparticles. First, there is a systematic and consistent improvement in increased birefringence and dielectric anisotropy. Secondly, it demonstrates that SPS nanoparticles, with their larger size and more wide distribution, give rise to stronger enhancement in the electro-optic parameters, as compared with $\mathrm{BaTiO}_{3}$. Dielectric anisotropy increased by up to $230 \%$ for the case of $\mathrm{BaTiO}_{3}$ nanoparticles and as much as up to $300 \%$ for the case of SPS nanoparticles. Similarly, the induced birefringence increased by up to $180 \%$ for $\mathrm{BaTiO}_{3}$ nanoparticles and up to $200 \%$ for SPS nanoparticles. Thirdly, the reorientation threshold is reduced by up to $50 \%$. Finally, increase in phase transition temperatures ranges from 5 to $12{ }^{\circ} \mathrm{C}$. For phase transition temperatures, the difference between the SPS and $\mathrm{BaTiO}_{3}$ nanoparticles appears to be less critical. By analyzing the temperature and electro-optic parameters of the liquid crystals functionalized with nanoparticles, we could derive some qualitative conclusions about the underlying processes. According to the recently developed theory, the main factors contributing to the magnitude of birefringence are the order parameter and polarizability. ${ }^{16}$ The expression describing the changes in birefringence $\Delta n$ can be written as

$$
\frac{\Delta n^{\text {func }}}{\Delta n^{\mathrm{LC}}}=\frac{n_{e}^{\mathrm{LC}}+n_{o}^{\mathrm{LC}}}{n_{e}^{\text {func }}+n_{o}^{\text {func }}} \frac{S_{\text {func }}}{S_{\mathrm{LC}}} \frac{\gamma^{\text {func }}}{\gamma^{\mathrm{LC}}},
$$

where $\Delta n^{\text {func }}$ is the birefringence of liquid crystals functionalized with nanoparticles, $\Delta n^{\mathrm{LC}}$ is the birefringence of liquid crystal, $n_{e}$ and $n_{o}$ are extraordinary and ordinary refractive

TABLE I. Comparison of dielectric, optical anisotropy, reorientation threshold, and phase transition temperatures for pure liquid crystals and liquid crystals with ferroelectric nanoparticles.

\begin{tabular}{lllll}
\hline \hline & $\Delta \varepsilon$ & $\Delta n$ & $V_{\mathrm{TH}}(\mathrm{V})$ & $T\left({ }^{\circ} \mathrm{C}\right)$ \\
\hline $\mathrm{LC} 18523_{\mathrm{LC} 18523+\mathrm{BaTiO}_{3}}$ & 2.1 & 0.05 & 1.95 & 57 \\
$\mathrm{LC} 18523+\mathrm{SPS}_{\mathrm{LC} 1550}$ & 6.8 & 0.09 & 1.05 & 68 \\
$\mathrm{LC} 1550+\mathrm{BaTiO}$ & 0.10 & 0.85 & 69 \\
LC1550+SPS & 2.38 & 0.05 & 1.45 & 79 \\
\hline \hline
\end{tabular}


indices of pure and functionalized liquid crystals, $S$ describes the order parameter for either pure or functionalized liquid crystals, and $\gamma$ refers to molecular polarizability anisotropy: $\gamma^{\mathrm{LC}}$ that of pure liquid crystals and $\gamma^{\text {func }}$ for their functionalized version, namely including the contribution from polarizability anisotropy of ferroelectric nanoparticles.

Each term of this equation can then be analyzed. The first term, the ratio of refractive indices, was measured and found to be constant. ${ }^{16}$ Furthermore, the second term relates to order parameters and, therefore, will be affected by changes in phase transition temperatures. As there is only relatively modest increase in phase transition temperatures in functionalized liquid crystals, it can be concluded that the ordering of liquid crystal molecules is not strongly affected by the presence of nanoparticles. As a result, the only remaining factor is the contribution from polarizability. It can be argued that it is the high polarizability of ferroelectric nanoparticles that contributes to increased birefringence. The two liquid crystals used are mixtures of several components, but both include hydrocarbon as well as fluorinated components. ${ }^{17}$ The consistent experimental results obtained in both LC 1550 and LC 18523, suggest that when nanoparticles are added, the effective polarizability anisotropy of functionalized liquid crystals increases as compared with their undoped version.

In conclusion, we demonstrated consistent and large improvement in electro-optic properties of the nematic, low refractive index liquid crystals doped with ferroelectric nanoparticles. The experimental investigation presented here demonstrates the feasibility of postsynthesis optimization of nematic liquid crystals, in particular their optical performance, via functionalization with inorganic nanoparticles.
The authors are very grateful to Yuri Reznikov, Gary Cook, Dean Evans, and Victor Reshetnyak for useful discussions and Alexander Grabar for advice and synthesis of SPS.

${ }^{1}$ I. C. Khoo, K. Chen, and Y. Z. Williams, IEEE J. Sel. Top. Quantum Electron. 12, 443 (2006).

${ }^{2}$ I. C. Khoo, D. H. Werner, X. Liang, A. Diaz, and B. Weiner, Opt. Lett. 31, 2592 (2006).

${ }^{3}$ P. N. Prasad, Curr. Opin. Solid State Mater. Sci. 8, 11 (2004).

${ }^{4}$ Q. Chen, M. R. Lin, J. E. Lee, Q. M. Zhang, and S. Yin, Appl. Phys. Lett. 89, 141121 (2006).

${ }^{5}$ F. Aslam, J. Stevenson-Hill, D. J. Binks, S. Daniels, N. L. Pickett, and P. O'Brien, Chem. Phys. 334, 45 (2007).

${ }^{6}$ J. H. Park and O. O. Park, Appl. Phys. Lett. 89, 193101 (2006).

${ }^{7}$ Y. Reznikov, O. Buchnev, O. Tereshchenko, V. Reshetnyak, A. Glushchenko, and J. West, Appl. Phys. Lett. 82, 1917 (2003).

${ }^{8}$ O. Buchnev, A. Dyadyusha, M. Kaczmarek, V. Reshetnyak, and Y. Reznikov, J. Opt. Soc. Am. B 24, 1512 (2007).

${ }^{9}$ F. Li, J. West, A. Glushchenko, and Ch.-I. Cheon, J. Soc. Inf. Disp. 14, 523 (2006).

${ }^{10}$ G. Cook, A. V. Glushchenko, V. Yu. Reshetnyak, E. R. Beckel, M. A. Saleh1, and D. R. Evans, IEEE/LEOS Winter Topical Meeting 2008 (unpublished), IEEE Catalog No. CFP08WTMC, p. 129.

${ }^{11}$ A. V. Gomonnai, Yu. M. Azhniuk, Yu. M. Vysochanskii, A. A. Kikineshi, M. Kis-Varga, L. Daroczy, I. P. Prits, and I. M. Voynarovych, J. Phys.: Condens. Matter 15, 6381 (2003).

${ }^{12}$ L. Huang, Z. Chen, J. D. Wilson, S. Banerjee, R. D. Robinson, I. P. Herman, R. Laibowitz, and S. O'Brien, J. Appl. Phys. 100, 034316 (2006).

${ }^{13}$ Y. Lin, J. Zhang, E. Kumacheva, and E. H. Sargent, Science 39, 993 (2004).

${ }^{14}$ S.-T. Wu, U. Efron, and L. D. Hess, Appl. Opt. 23, 3911 (1984).

${ }^{15}$ G. Cook, J. L. Carns, M. A. Saleh, and D. R. Evans, Mol. Cryst. Liq. Cryst. 453, 141 (2006).

${ }^{16} \mathrm{~F}$. Li, O. Buchnev, C. I. Cheon, A. Glushchenko, V. Reshetnyak, Yu. Reznikov, T. J. Sluckin, and J. L. West, Phys. Rev. Lett. 97, 147801 (2006).

${ }^{17}$ Y.-P. Lan, Y.-F. Lin, Y.-T. Li, R.-P. Pan, Ch.-K. Lee, and C.-L. Pan, Opt. Express 13, 7905 (2005). 